\title{
A Comparative Study of Narrowband Microcellular Speech Transmission Schemes
}

John E. B. Williams, Lajos Hanzo, Senior Member, IEEE, Raymond Steele, Senior Member, IEEE, and Joseph C. S. Cheung

\begin{abstract}
Experiments are conducted on a number of mobile radio systems that are suitable for operation in nondispersive microcellular networks. It becomes apparent that the selection of the type of modem and the number of its levels, the choice of speech codec to be used, and whether to deploy a channel codec, depends on the channel signal-to-noise ratio (SNR) and teletraffic demand, if the power consumption of the hand held portable is to be minimized. Following the implications of our limited number of experiments, we generalize our findings to some of the requirements of an adaptive transceiver that might be deployed in the next generation of mobile radio networks.
\end{abstract}

\section{INTRODUCTION AND SYSTEM OVERVIEW}

$\mathbf{T}$ HERE IS much activity worldwide in attempting to define the third generation mobile personal communications network (PCN). In the European RACE II program, there are two projects dealing with multiple access methods, one is concerned with code division multiple access (CDMA) and the other with time division multiple access (TDMA). From these studies, we expect to have the information to resolve the type of multiple access method for the universal mobile telecommunications system (UMTS), which the Europeans intend to design by the turn of the century. Somewhere along the line, UMTS is expected to merge with the CCIR study on the future public land mobile telecommunications system (FPLMTS).

In designing a third generation system, cognizance is given to the second generation systems. Indeed, we may anticipate that some of the subsystems of GSM and DECT may find their way into UMTS, either as a primary subsystem, or as a component to achieve backward compatibility with systems in the field. This approach may result in hand-held transceivers that are intelligent multimode terminals, able to communicate with existing networks, while having more advanced and adaptive features that we would expect to see in the next generation personal communication networks.

In this text, we present the results of a series of experiments we conducted. We concerned ourselves with the transceiver

Manuscript received April 12, 1993; revised August 24, 1993. This work was supported by the Radiocommunications Agency.

J. E. B. Williams is with Multiple Access Communications Limited, Southampton, S09 5NH, England, UK.

L. Hanzo and J. C. S. Cheung are with the University of Southampton, Department of Electronics and Computer Science, Southampton, S09 5NH England, UK.

R. Steele is with the University of Southampton, Department of Electronics and Computer Science and Multiple Access Communications Limited, Southampton, S09 5NH, England, UK.

IEEE Log Number 9403811. structure depicted in Fig. 1. The Voice Activity Detector (VAD) [1] detects, whether active speech or only acoustic background noise is at the speech encoder's input. If an active speech spurt is to be encoded and transmitted, the transceiver is enabled and the speech packet is queued by the packet reservation multiple access (PRMA) [2] slot allocator for reserving a time slot for future uncontended use. If no reservation is granted within some $30 \mathrm{~ms}$, the speech packet must be dropped, but the dropping probability must be kept below $1 \%$ for state-of-the-art speech codecs in order to prevent speech impairments. Should the input signal be deemed to be noise by the VAD, the transceiver can be switched into its dormant mode in order to save battery power.

In the friendly low dispersion, high signal-to-noise ratio (SNR) cordless telecommunications (CT) environment [3], [4, ch. 1, p. 76], the speech coded bits are typically unprotected in order to minimize the system's complexity and battery drain. In more robust schemes, source sensitivity matched kclass forward error correction (FEC) arrangements are favored [5]-[7]. Accordingly, the bits are mapped by the Bit Mapper of Fig. 1 to the input of the appropriate FEC encoder, where FECk is assumed to be the most powerful one, protecting the most vulnerable source bits. An intelligent transceiver could also facilitate the deployment of variable rate embedded FEC schemes, varying the FEC coding power depending on the availability of more PRMA slots under lightly loaded network conditions. The FEC protected speech bits are then modulated onto a $2 \mathrm{GHz}$ radio carrier and transmitted via the nondispersive, flat Rayleigh fading microcellular channel. At the receiver, after automatic gain control (AGC), the side information representing the type of modulation scheme and other system characteristics is extracted and the inverse operations are carried out in order to recover the original speech. The error detection capability of the most powerful codec, FECk, can be used to initiate handovers or to detect corrupted speech segments and enhance their subjective quality [9].

In this comparative study, we introduced a menu that includes some of the subsystems that are to be found in the second generation mobile radio networks, such as the $900 \mathrm{MHz}$ Pan-European system, known as GSM [4, ch. 8 , p. 677] or the $2 \mathrm{GHz}$ British DCS 1800 scheme, the American IS-54 [12], the Japanese digital mobile radio system [13], and the low-complexity Digital European Cordless Telecommunications (DECT) or the CT2 schemes [4, ch. 1, p. 79]. These arrangements may also be incorporated in the future third generation systems due to the need for 


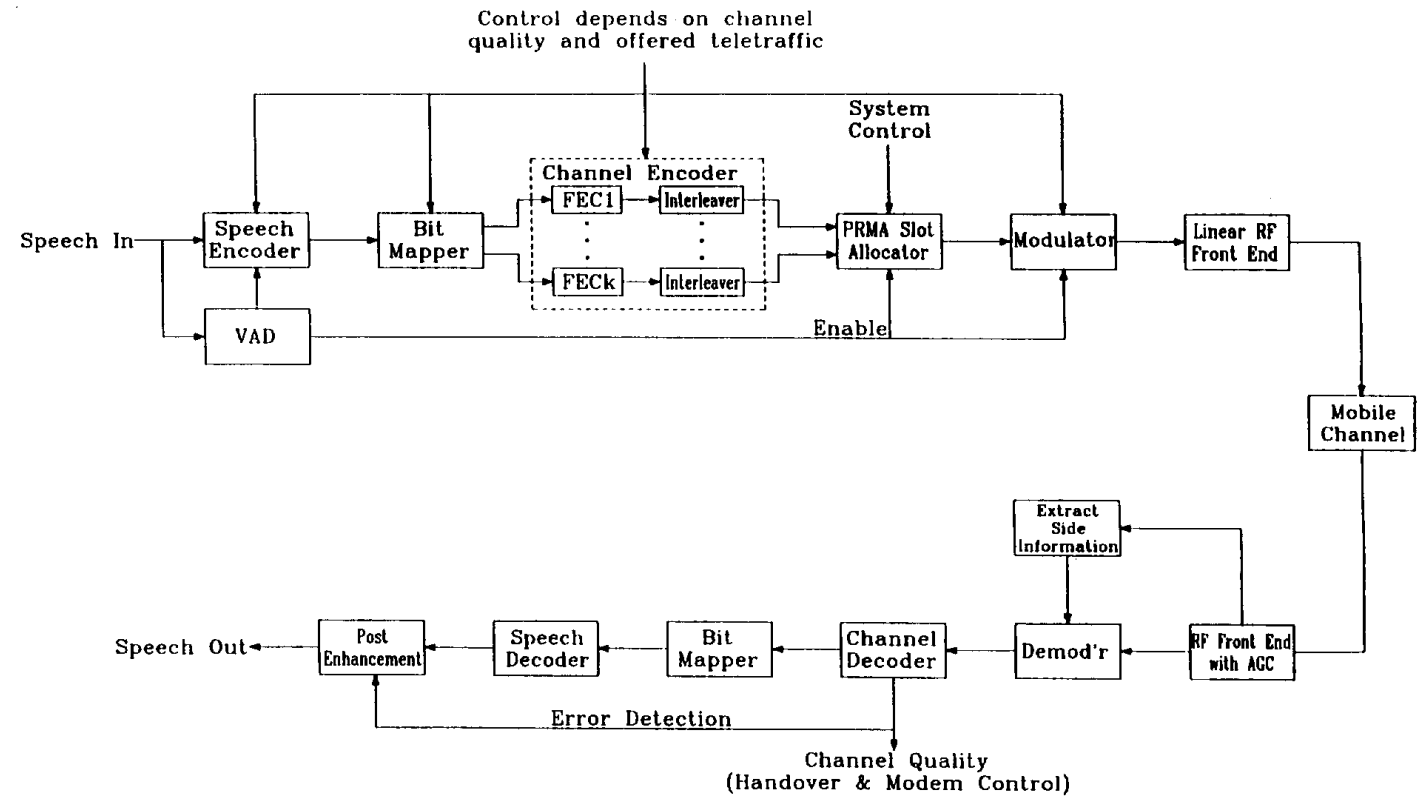

Fig. 1. Transceiver architecture.

backward compatibility. Additionally, we included $4 \mathrm{~b} / \mathrm{symbol}$ multilevel modulation [10], as we consider that this bandwidth efficient but vulnerable scheme can be made more robust by reconfiguring it under system control, for example, as $\frac{\pi}{4}$-Shifted Differential Quadrature Phase Shift Keying, $\frac{\pi}{4}$ DQPSK, and Gaussian Minimum Shift Keying (GMSK) when the channel quality degrades. Multilevel modulation also has attractive properties when used in packet reservation multiple access (PRMA), as we will highlight. In order to limit the variety of scenarios and ensure low complexity light weight construction, only low power drain speech codecs, such as the $32 \mathrm{~kb} / \mathrm{s}$ Adaptive Differential Pulse Code Modulated (ADPCM) codec of the CT2 or DECT systems and the 13 $\mathrm{kb} / \mathrm{s}$ Regular Pulse Excited (RPE) GSM speech codec [4, ch. 8, p. 700] are considered.

The two speech codecs combined with the three modems yield six transceiver schemes. However, with each modem constellation, we used both a low complexity and a high complexity detector, in order to explore the complexityperformance trade-offs. The resulting 12 transceivers denoted by A-L are listed in Table I. Details for columns 3-14 of Table I will be revealed at a later stage. The performance of these transceivers was evaluated using PRMA and an identical modulation signalling rate of $400 \mathrm{kBd}$ over both Gaussian and nondispersive $2 \mathrm{GHz}$ Rayleigh fading channels in order to gauge the achieveable performance limit, when using an adaptive transceiver incorporating the firmware of systems A-L.

The identical Baud rate was selected in order to ensure a fair comparison of all modulation schemes by exposing them to identical fading rates. Although this results in a different throughput due to the variable number of bits/symbol and different multiuser bandwidth due to the modems' different filtering requirements, this is taken into account when computing the actual PRMA user bandwidths derived later in Section VII-C and given in column 12 of Table I. Another feasible principle that could be pursued in our comparisons would be to use different signalling rates for the different modems while maintaining the same PRMA system bandwidth requirement. However, this would provide different propagation conditions for all modems and distort the robustness figures while still yielding different source throughput rates.

This paper is organized as follows. In Sections II, III and IV, we consider radio modems, speech codecs and channel codecs, respectively. Armed with a set of these subsystems, we construct a menu of speech transmission systems in Section V. In Section VI, we discuss PRMA. Section VII discusses the simulation results of our candidate speech links, enabling us to describe in Section VIII how an intelligent terminal could adapt itself in different microcellular environments.

\section{MODEM SCHEMES}

The choice of modem is based on the interplay of equipment complexity, power consumption, spectral efficiency, robustness against channel errors, cochannel and adjacent channel interference, as well as the propagation phenomena, which depends on the cell size [4]. Equally important are the associated issues of linear or nonlinear amplification and filtering, the applicability of noncoherent, differential detection, soft decision detection, equalization, and so forth. Here, we briefly describe GMSK modulation [4, ch. 6, p. 523], which is used in the European second generation systems, $\frac{\pi}{4}$-DQPSK modulation favored by the Americans in their IS-54 D-AMPS network [12] and by the Japanese digital cellular system [13], and 16-level star QAM [10], which works well in microcellular environments. 
TABLE I

SYSTEM COMPARISON

\begin{tabular}{|c|c|c|c|c|c|c|c|c|c|c|c|c|c|}
\hline \multirow[t]{2}{*}{$\begin{array}{c}1 \\
\text { System }\end{array}$} & \multirow[t]{2}{*}{$\begin{array}{c}2 \\
\text { Modulator }\end{array}$} & \multirow[t]{2}{*}{$\begin{array}{c}3 \\
\text { Delector }\end{array}$} & \multirow[t]{2}{*}{$\begin{array}{c}1 \\
\text { FEC }\end{array}$} & \multirow{2}{*}{$\begin{array}{c}5 \\
\text { Speech } \\
\text { Cndec } \\
\end{array}$} & \multirow{2}{*}{$\begin{array}{c}6 \\
\text { Complexity } \\
\text { Order }\end{array}$} & \multirow{2}{*}{$\begin{array}{c}7 \\
\text { Haud Kate } \\
(K \mid 3,1)\end{array}$} & \multirow{2}{*}{$\begin{array}{c}\text { B } \\
\text { TDMA User } \\
\text { Bandw. (kIlz) }\end{array}$} & \multirow{2}{*}{$\begin{array}{c}9 \\
\text { No of 'l'DMA } \\
\text { Uscrs/Carrier }\end{array}$} & \multirow{2}{*}{$\begin{array}{c}10 \\
\text { No of PRMA } \\
\text { Users/Carricr }\end{array}$} & \multirow{2}{*}{$\begin{array}{c}\| 1 \\
\text { No of PRMA } \\
\text { Uscrs/siot }\end{array}$} & \multirow{2}{*}{$\begin{array}{c}12 \\
\text { PRMA User } \\
\text { [Randw. (kHz) }\end{array}$} & \multicolumn{2}{|c|}{\begin{tabular}{c|c}
13 & 14 \\
$\operatorname{Min}$ & SNR $(\mathrm{dB})$ \\
\end{tabular}} \\
\hline & & & & & & & & & & & & AWGN & Rayleigh \\
\hline A & GMSK & Viterbi & No & ADPCM & 2 & 32 & 23.7 & 11 & 18 & 1.64 & 14.5 & 7 & $\infty$ \\
\hline B & GMSK & Freq. Discr. & No & ADPCM & 1 & 32 & 23.7 & 11 & 18 & 1.64 & 14.5 & 21 & 31 \\
\hline $\mathrm{c}$ & $\frac{\pi}{i}$-DQPSK & MLH-CR & No & ADPCM & 4 & 16 & 19.8 & 22 & 42 & 1.91 & 10.4 & 10 & 28 \\
\hline D & $\frac{\pi}{7}$-DQPSK & Differential & No & ADPCM & 3 & 16 & 19.8 & 22 & 42 & 1.91 & 10.4 & 10 & 28 \\
\hline E & I6-StQAM & MLLIf-CR & $\mathrm{Na}$ & $A D P C M$ & 6 & 8 & 13.3 & 44 & 87 & 1.98 & 6.7 & 20 & $\infty$ \\
\hline $\mathbf{F}$ & 16-StQAM & Differential & No & ADPCM & 5 & 8 & 13.3 & 44 & 87 & 1.98 & 6.7 & 21 & 31 \\
\hline G & GMSK & Vitcrlji & BCII & RPE-LTP & 8 & 24.8 & 18.4 & 12 & 22 & 1.83 & 10.1 & 1 & 15 \\
\hline II & GMISK & Frey. Disct. & $\mathrm{BCH}$ & RPE-LTP & 7 & 24.8 & 18.4 & 12 & 22 & 1.83 & 10.1 & 8 & 18 \\
\hline 1 & T-DQPSK & MLH-CR & $\mathrm{BCH}$ & RPE-LTP & 10 & 12.4 & 15.3 & 24 & 46 & 1.92 & $B$ & 5 & 20 \\
\hline J & $\frac{\pi}{f}$ DQPSK & Differential & $\mathrm{BCH}$ & RPE.LTP & 9 & 12.4 & 15.3 & 24 & 46. & 1.92 & 8 & 6 & 18 \\
\hline $\mathrm{K}$ & 16-StQAM & MLII-CR & $\mathrm{BCH}$ & RPE-LTP & 12 & 6.2 & 10.3 & 48 & 96 & 2.18 & 4.7 & 13 & 25 \\
\hline $\mathbf{L}$ & 16-StQAM & Ditferential & $\mathrm{BCH}$ & RPE-LTP & 11 & 6.2 & 10.3 & 48 & 96 & 2.18 & 4.7 & 16 & 24 \\
\hline
\end{tabular}

GMSK: Gaussian Minimum Shift Keying

$\frac{\pi}{4}$-DQPSK: Differential Phase Shift Keying

16-StQAM: 16-level Quadrature Amplitude Modulation

MLH-CR: Maximum Likelihood Correlation Receiver

BCH: Bose Chaudhuri Hoequenghem FEC Coding

ADPCM: Adaptive Differential Pulse Code Modulation

RPE-LTP: Regular Pulse Excited Speech Codec with Long-Term Prediction

TDMA: Time Division Multiple Access

PRMA: Packet Reservation Multiple Access

\section{A. GMSK Modulation}

In Gaussian Minimum Shift Keying (GMSK), the phase transitions are determined as the convolution of a Gaussian pulse shaping function $g(t)$ with the symbol sequence [14]. If $B_{b}$ is the bandwidth of a low pass filter having a Gaussian shaped spectrum and $T$ is the bit period, then $B_{N}=B_{b} T$ is the normalized bandwidth.

Since the length of the Gaussian pulse exceeds the signalling interval, response of the modulator to a symbol is spread over several symbol periods introducing controlled intersymbol interference (CISI), which curtails the bandwidth of the modulated signal. If the phase transitions are unfiltered, $B_{N}=\infty$ and we have a Minimum Shift Keying (MSK) modulator. As $B_{N}$ is reduced, more and more CISI occurs, but the out-of-band spectral spillage is reduced correspondingly. As examples of typical GMSK modulators, the Pan-European system, GSM uses a GMSK modulator with $B_{N}=0.3$, which requires sophisticated equalization to achieve good performance. The digital European cordless telecommunications (DECT) system's modulator uses $B_{N}=0.5$, which eases clock recovery. The typical spectral occupancy, in terms of bits per $\mathrm{Hz}$ of a GSM-like GMSK modem, is 1.35 .

As $B_{N}$ is reduced, the spectrum becomes more compressed but the phase transitions follow a smoother curve and more CISI is introduced, rendering bit-by-bit detection using a frequency discriminator more prone to channel impairments. Maximum Likelihood Sequence Estimation (MLSE) using the Viterbi algorithm [4] is more efficient in removing both CISI and ISI introduced by the channel, at the cost of increased complexity. Furthermore, with decreasing $B_{N}$, the eye-pattern becomes more complicated and the reduced eyeopening makes clock and carrier recovery more difficult to achieve.

Full response CPM schemes, such as MSK, can be demodulated by a frequency discriminator, if the ISI is not excessive, by simply measuring the change of phase over a symbol period. When the phase increases, its derivative, the frequency, is positive and a logical " 1 " is detected and vice versa when the phase decreases.

If the discriminator is oversampled, it can demodulate GMSK signals [15] having appreciable spreading but with degraded noise margins. As the discriminator operates by differentiating the received signal, it is important to deploy a preceding lowpass filter (LPF) to smooth the phase perturbations caused by the noise. Otherwise, the performance at low channel signal-io-noise ratios (SNR's) is seriously affected, where the channel SNR is defined as the ratio of the average received signal power and noise power. For a more detailed treatise on GMSK consult [4, ch. 6, p. 523].

\section{B. $\frac{\pi}{4}-D Q P S K$ Modulation}

A particular form of differential quadrature phase shift keying, known as $\frac{\pi}{4}$-DQPSK [8], transmits 2 b/symbol, allowing the bandwidth to be reduced at the cost of increased complexity and degraded SNR and signal-to-interference ratio (SIR) performances. Careful filtering and linear amplification of the modulated signal are required to achieve good adjacent channel performance. Because there are $2 \mathrm{~b} / \mathrm{symbol}$, the bit rate can be higher than with GMSK before it is necessary to use a channel equalizer.

In this linear modulation, the amplitude variations are reduced compared to conventional DQPSK due to the rotation of the constellation by $\frac{\pi}{4}$ every symbol period, ensuring that no phasor transition path passes through the zero amplitude point. The data stream is differentially Gray encoded in order to allow low complexity differential detection to be employed, 
where the Gray coding ensures that decoding into the adjacent erroneous symbol corrupts one bit only. For a more detailed treatise on $\frac{\pi}{4}$-DQPSK refer to [12], [13], [8].

Alternatively, multiple-symbol differential detection [16], [17] using the MLSE Viterbi algorithm can be implemented to achieve better performance at higher complexity. The algorithm implemented allows using an arbitrarily long trace-back memory, but in order to limit the complexity of the multilevel Viterbi detector, we restricted its computation window to one signalling interval when it becomes essentially a maximum likelihood (MLH) correlation receiver (MLH-CR). Decisions are carried out using coherent detection and all possible filtered, 16-times oversampled transmitted waveforms are compared with the received one, in order to identify the most likely transmitted one associated with the lowest Euclidean distance.

In our scheme, 129 th order Blackman-windowed root raised cosine finite impulse response (FIR) Nyquist transmit and receive filters with a roll-off factor of $\alpha=0.35$ are employed and an equalizer is only required when operating in a wideband environment. The $\frac{\pi}{4}$-DQPSK spectral occupancy is 1.62 $\mathrm{b} / \mathrm{Hz}$, defining the transmission bandwidth as the spectral separation where the spectrum is $24 \mathrm{~dB}$ below its value at the carrier. This value of $24 \mathrm{~dB}$ is considerably higher than that required for similar adjacent channel protection in GMSK modems.

This differentially encoded scheme is readily demodulated by a noncoherent differential detector by simply evaluating the phasor changes between adjacent sampling instants. Since coherent carrier recovery is not necessary, reduced complexity is achieved and carrier recovery false locking problems are eliminated. However, at low SNR and SIR values, some performance penalty is incurred. Differential phase detection schemes are also applicable for demodulation of combined amplitude shift keying (ASK) constellations such as quadrature amplitude modulation (QAM).

\section{16-StQAM Modem}

The well-known square shaped 16-QAM phasor constellation is optimum, in terms of bit error rate (BER), for transmissions over Gaussian channels due to its maximal minimum Euclidean distance amongst its constellation points [9], when compared to other 16-level constellations. However, over Rayleigh fading channels, its performance is impaired by occasional false locking of its carrier phase.

For fading channels, the differentially coded Star QAM (StQAM) scheme proposed in [10] has a better performance than square QAM in spite of the error-doubling property of its differential coding. Indeed, when using noncoherent demodulation, the penalty in terms of SNR is lower than the expected 3 $\mathrm{dB}$. Therefore, we opted for this 16-StQAM scheme, where the first bit of a four-bit symbol $b_{1}$ is differentially encoded onto the twin rings of the phasor constellation, while the remaining three bits are differentially Gray-coded onto the eight phase positions. The two rings are at radii of $A_{1}$ and $A_{2}$.

Using the above differential encoding technique, decoding data is now reduced to a comparison test between the previous and current received symbols. Let the received phasor amplitudes be $Z_{t}$ and $Z_{t+1}$ at time $t$ and $t+1$, respectively. The demodulator must identify whether there has been a significant change in amplitude in order to, regenerate a logical " 1 ." This is equivalent to asking whether the amplitude of the phasor at the transmitter changed rings at time $t+1$ compared to time $t$. The algorithm employed at the demodulator uses two adaptive thresholds to carry out its decisions. If

$$
Z_{t+1} \geq\left(\frac{A_{1}+A_{2}}{2}\right) Z_{t}
$$

or if

$$
Z_{t+1}<\left(\frac{2}{A_{1}+A_{2}}\right) Z_{t}
$$

then a significant change in amplitude is deemed to have occurred, and bit $b_{1}$ is set to logical " 1 " at time $t+1$. Notice that the thresholds are dependent on $Z_{t}$, and as the amplitude of the phasors change in fading conditions, so do the thresholds. Should both inequalities (1) and (2) fail to be satisfied, $b_{1}$ is assigned logical " 0. ."

If the received symbol phases are $\theta_{t}$ and $\theta_{t+1}$ at time $t$ and $t+1$, respectively, the demodulated angle is

$$
\theta_{\text {dem }}=\left(\theta_{t+1}-\theta_{t}\right) \bmod 2 \pi \text {. }
$$

This angle is then quantized to the nearest multiple of 45 degrees and a lookup table is consulted in order to derive the remaining three output bits, $b_{2}, b_{3}$ and $b_{4}$.

Spectral compactness is achieved by the $129 t h$ order Blackman-windowed root raised cosine FIR Nyquist transmit and receive filtering with a roll-off factor of $\alpha=1$. With this proviso, the 16-StQAM bandwidth occupancy is $2.4 \mathrm{~b} / \mathrm{Hz}$, defining the transmission bandwidth as the spectral separation, where the spectrum is $24 \mathrm{~dB}$. below its value at the carrier. When using an oversampling ratio of 16 , the filtering delay is four signalling intervals. As with $\frac{\pi}{4}$-DQPSK, the performance of this differential detection will also be compared to that of MLH-CR.

\section{SPEECH CODECS}

In recent years, both speech coding research and ICtechnology went through a revolutionary development, culminating in a number of standards with bit rates as low as $4.8 \mathrm{kbps}$ [4]. The choice of an appropriate speech codec is based on the complex interplay of speech quality, computational complexity related to power consumption, bit rate, delay and robustness against channel errors.

The two speech codecs used in our experiments are the CCITT G 721. 32 kbps adaptive differential pulse code modulation (ADPCM) codec used in the DECT system, and the 13 kbps GSM and DCS 1800 Regular Pulse Excited (RPE), Long Term Predictor (LTP) assisted codec. The ADPCM codec used in our schemes is well known so we will not describe its operation here. We point out that, when transmitting ADPCMcoded speech, the bit-sensitivity within each four-bit symbol monotonically decreases from the Most Significant Bit (MSB) towards the Least Significant Bit (LSB). 
Our RPE-LTP codec is a modified version of the standard scheme [11] because by using ten Line Spectral Frequencies (LSF) instead of the standardized eight Logarithmic Area Ratios (LAR's), we obtain a slightly better performance when encoding them with $36 \mathrm{~b}$. This is mainly due to their socalled ordering property [4], implying that the LSF parameters are monotonically increasing with increasing parameter index. This property allows the detection of channel errors that violate the ordering property, and the situation can be improved by LSF extrapolation. A further difference in our RPE-LTP codec is that we use four bits to encode the LTP gain instead of the standard two bits. This results in significant speech quality improvement. Therefore, the total number of bits is 268 per $20 \mathrm{~ms}$ frame and the overall bit rate is increased to $13.4 \mathrm{kbps}$.

For robustness against channel errors some speech bits are better protected than others. To identify the effect of bit errors on speech quality we performed a bit sensitivity analysis based on a combination of Segmental SNR (SSNR) and Cepstral Distance (CD) degradation, since different objective speech quality measures quantify different types of degradations [19]. The SSNR is a wave form fidelity measure, while the CD measure quantifies the speech spectral envelope distortion. Our findings are presented in [11]. In order to verify the subjective relevance of our combined objective measures, we compared our findings with the subjective test results published in the GSM Recommendations. Our analysis yielded a sensitivity order very similar to those found by GSM, verifying our approach. For the sake of low complexity, a simple twin class error protection scheme is used where the more important bits are termed as Class 1 (C1) bits and the less sensitive ones as Class 2 (C2) bits. Slight variations in the sensitivity order of bits within Class 1 or Class 2 do not affect the system performance.

In comparison, the $32 \mathrm{kbps}$ ADPCM waveform codec has a segmental SNR (SSNR) of about $28 \mathrm{~dB}$, while the 13 kbps analysis-by-synthesis (ABS) RPE-LTP codec has a lower SSNR of about $16 \mathrm{~dB}$, associated with similar subjective quality rated as a mean opinion score (MOS) of about four. This discrepancy in SSNR is because the RPE-LTP codec utilizes perceptual error weighting. The cost of the RPELTP codec's significantly lower bit rate and higher robustness compared to ADPCM is its increased complexity and encoding delay.

\section{Channel CODING AND BIT-MAPPING}

In mobile communications, both convolutional and block codes having different strengths and weaknesses are deployed [4].

In the higher complexity, more robust, lower bit rate speech transmission systems based on the RPE-LTP codec, we favor binary Bose-Chaudhuri-Hocquenghem (BCH) block codes. They combine low computational complexity with high error correcting power, even when using hard decisions. They also have reliable error detection properties, which are used by our speech postenhancement scheme [9], when the received speech is corrupted due to an FEC decoding failure. The channel codec may also be employed to assist in initiating fast handovers, which are vitally important in small microcells, where they may occur frequently and fast.

In our twin-class FEC scheme [11], the $116 \mathrm{Cl}$ bits of the RPE-LTP codec are protected by a shortened binary BCH $(62$, 29,6 ) code yielding $248 \mathrm{~b}$, while the $152 \mathrm{C} 2 \mathrm{~b}$ are coded by a shortened binary BCH $(62,38,4)$ code which also yields 248 b. Four 62-b codewords are used in each class, which are rectangularly interleaved to curtail error propagation across $20 \mathrm{~ms}$ speech frames. The $496 \mathrm{~b}$ in one speech frame are transmitted during $20 \mathrm{~ms}$, yielding a total transmission bit rate of $24.8 \mathrm{~kb} / \mathrm{s}$.

\section{SPEECH TRANSMISSION SYSTEMS}

In our simulations, we used the GMSK, $\frac{\pi}{4}$-DQPSK and 16-StQAM modems combined with both the unprotected lowcomplexity $32 \mathrm{kbps}$ ADPCM codec (as in DECT and CT2) and the $13 \mathrm{kbps}$ RPE-LTP codec with its FEC. Each modem had the option of either a low or a high complexity demodulator. Synchronous transmissions and perfect channel estimation were assumed in evaluating the relative performances of the systems listed in Table I. Our results represent performance upper bounds, allowing relative performance comparisons under identical cicumstances. The propagation environment was assumed to be Gaussian, or Rayleigh, which is the worst type of fading in microcells. We presumed that the cells were sufficiently small, for the transmitted symbol rate considered that dispersion in the radio channels was a rare event.

Returning to Table I, the first column shows the system classification letter, the next the modulation used, the third the demodulation scheme employed, the fourth the FEC scheme and the fifth the speech codec deployed. The sixth column gives the estimated relative order of the complexity of the schemes, where the most complex one having a complexity parameter of 12 is the 16-StQAM, MLH-CR, BCH, RPELTP arrangement. All the BCH-coded RPE-LTP schemes have complexity parameters larger than six, while the unprotected ADPCM systems are characterized by values of one to six, depending on the complexity of the modem used. The speech Baud rate and the TDMA user bandwidth are given next. Observe that the DQPSK and 16-StQAM Baud rate figures have been moderated by the actual bandwidth requirements computed by taking into account their filtering requirements. Explicitly, the $1 \mathrm{~b} / \mathrm{symbol}$, GMSK, $2 \mathrm{~b} / \mathrm{symbol} \frac{\pi}{4}$-DQPSK and $4 \mathrm{~b} /$ symbol 16-StQAM modems have moderated spectral occupancy figures of $1.35,1.62$ and $2.4 \mathrm{~b} / \mathrm{Hz}$, respectively. A signalling rate of $400 \mathrm{kBd}$ was chosen for all our experiments, irrespective of the number of modulation levels, to provide a fair comparison for all the systems, under identical propagation conditions. The corresponding $400 \mathrm{kBd}$ systems have a total bandwidth of $400 / 1.35=296 \mathrm{kHz}, 2 \cdot 400 / 1.62=494 \mathrm{kHz}$ and $4 \cdot 400 / 2.4=667 \mathrm{kHz}$, respectively.

The last two columns, columns 13 and 14, list the minimum channel SNR values in $\mathrm{dB}$ required for the system to guarantee nearly unimpaired speech quality through Gaussian and Rayleigh channels. These are characteristics, closely related to the system's robustness, and they will be derived later, after Section VII. 
TABLE II

Summary of Prma Parameters

\begin{tabular}{|l|r|}
\hline PRMA parameter & $400 \mathrm{kBd}$ \\
\hline Channel rate & variable (Table 1, col. 7) \\
Source rate & $20 \mathrm{~ms}$ \\
Frame duration & variable (Table 1, col. 9) \\
No. of slots = TDMA users/carrier & variable (20 ms/No. of slots) \\
Slot duration & 64 bits \\
Header length & $32 \mathrm{~ms}$ \\
Maximum speech delay & variable \\
Permission probability & \\
\hline
\end{tabular}

\section{PaCKet Reservation Multiple AcCess}

PRMA is a convenient statistical multiplexing method for conveying speech signals via TDMA systems. A full treatment of PRMA is given in [2], [18]. The operation of PRMA is based on the voice activity detector (VAD) [1] being able to reliably detect inactive speech segments. Inactive users' TDMA time slots are allocated to other users, who become active. The users who are just becoming active have to contend for the available time slots with a certain permission probability, which is an important PRMA parameter. The reason for allowing previously colliding users to contend for the next available time slot only with a less than unity permission probability is to prevent them from consistently colliding in their further attempts to attain reservation. If more than one user is contending for a free slot, neither of them will be granted it. If, however, only one user requires the time slot, he can reserve it for future use until he becomes inactive. Under heavily loaded network conditions, when many users are contending for a reservation, a speech packet might have to contend for a number of consecutive slots. When the contention delay exceeds $32 \mathrm{~ms}$, the speech packet of $20 \mathrm{~ms}$ duration must be dropped. The probability of packet dropping must be kept below $1 \%$, a value inflicting minimal degradation in perceivable speech quality in contemporary speech codecs. More recent advanced speech codecs, such as the future lowdelay $8 \mathrm{~kb} / \mathrm{s}$ CCITT Code Excited Linear Predictive (CELP) codec can tolerate $20 \mathrm{~ms}$ packet dropping rates up to $2 \%$ with no apparent speech degradation.

Further important PRMA parameters are listed in Table II. Negative exponentially distributed speech spurts and speech gaps with mean durations of 1 and $1.35 \mathrm{~s}$ were used in the simulations. The transmitted Baud rate was fixed to $400 \mathrm{kBd}$, as before, while the source rate was variable, depending on the system used. The source Baud rates for systems A-L are listed in column 7 of Table I. The PRMA frame duration was $20 \mathrm{~ms}$ and the number of PRMA slots was identical to the number of TDMA slots per frame or TDMA users per carrier.

The number of TDMA and PRMA slots per frame was computed from the frame duration as follows. During a PRMA frame of $20 \mathrm{~ms}$, the $32 \mathrm{kbps}$ ADPCM speech encoder generated $640 \mathrm{~b}$ of speech information. A header length of 64 b was assumed to serve signalling and control purposes and so the $704 \mathrm{~b} / 20 \mathrm{~ms}=35.2 \mathrm{kbps}$ source information rate allowed us to support integer of $400 / 35.2$, namely 11 time slots, i.e., 11 TDMA users per $400 \mathrm{kBd}$ bearer, in case of unprotected binary transmissions, such as in systems $\mathrm{A}$ and $\mathrm{B}$. When using the 2 and $4 \mathrm{~b} / \mathrm{Hz} \frac{\pi}{4}$-DQPSK and 16-StQAM modems, the packet length was decreased by factors of 2 and 4 , respectively, causing the number of slots per frame to increase by similar factors. Thus, the use of multilevel modulation increases the number of PRMA slots.

For the FEC protected scenarios, a $2 \times 29=58$-b long header was assumed. Each 29-b block in the header was BCH $(62,29,6)$ coded to give a header of $124 \mathrm{~b}$. The $20 \mathrm{~ms}$ PRMA frame was constituted by $496+124=620 \mathrm{~b}$, yielding a bit rate of $31 \mathrm{kbps}$. The number of PRMA slots supported in case of the 1,2 and $4 \mathrm{~b} / \mathrm{Hz}$ scenarios was 12,24 and 48 , respectively.

We compared the previously computed PRMA slot numbers with those when binary transmissions associated with halfand quarter-rate speech codecs were used. The $16 \mathrm{kbps}$ halfrate speech codec generated $320 \mathrm{~b} / 20 \mathrm{~ms}$ and after adding the header length of $64 \mathrm{~b}$, the transmission rate became 384 b/20 ms $=19.2 \mathrm{kbps}$, creating 20 PRMA slots. For an $8 \mathrm{kbps}$ quarter-rate speech codec, the information rate was $224 \mathrm{~b} / 20$ $\mathrm{ms}=11.2 \mathrm{kbps}$, accommodating 35 PRMA slots.

By using multilevel modems instead of half- or quarter-rate speech codecs, we were able to support more PRMA slots. This yielded further benefits in terms of reducing the packet dropping probability and thereby increased the number of users supported, i.e., the PRMA efficiency.

\section{PERFORMANCE COMPARISON}

The system performances apply to microcellular conditions. The carrier frequency was $2 \mathrm{GHz}$, the data rate $400 \mathrm{kBd}$, and the mobile speed $15 \mathrm{~m} / \mathrm{s}$. At $400 \mathrm{kBd}$ in microcells, the fading is flat (the transmission rate in DECT is 1152 $\mathrm{kBd}$ and even at this high rate it is not necessary to use FEC codecs or equalizers) and usually Rician. The best and worst Rician channels are the Gaussian and Rayleigh fading channels, respectively, and we performed our simulations for these channels to obtain upper and lower bound performances. Our conditions of $2 \mathrm{GHz}, 400 \mathrm{kBd}$ and $15 \mathrm{~m} / \mathrm{s}$ is arbitrary. They correspond to a fading pattern that can be obtained for a variety of different conditions, for example, at $900 \mathrm{MHz}, 271$ $\mathrm{kBd}$ and $23 \mathrm{~m} / \mathrm{s}$. Our goal is to compare the performances of the systems defined in Table I when operating according to our standard conditions.

\section{A. Modem Performance}

We examine the modem performance associated with systems A to F. We are only concerned in this subsection with the bit error rate (BER) versus channel SNR characteristics. In Section VII-B, we examine the performance of the systems when handling speech signals.

1) GMSK Performance: In our GMSK modem simulations, we considered narrowband systems similar to GSM or DCS 1800 at $15 \mathrm{~m} / \mathrm{s}$, and CT2 at $6 \mathrm{~m} / \mathrm{s}$, with $B_{T}=0.3$ and $B_{T}=0.5$, respectively. With the lower $B_{T}=0.3$ value, better spectral compactness was achieved with a concomitant increase in complexity, necessitating Viterbi decoding instead of the less complex frequency discriminator used in the DECT-like modem. Also, with $B_{T}=0.3$, the synchronization 


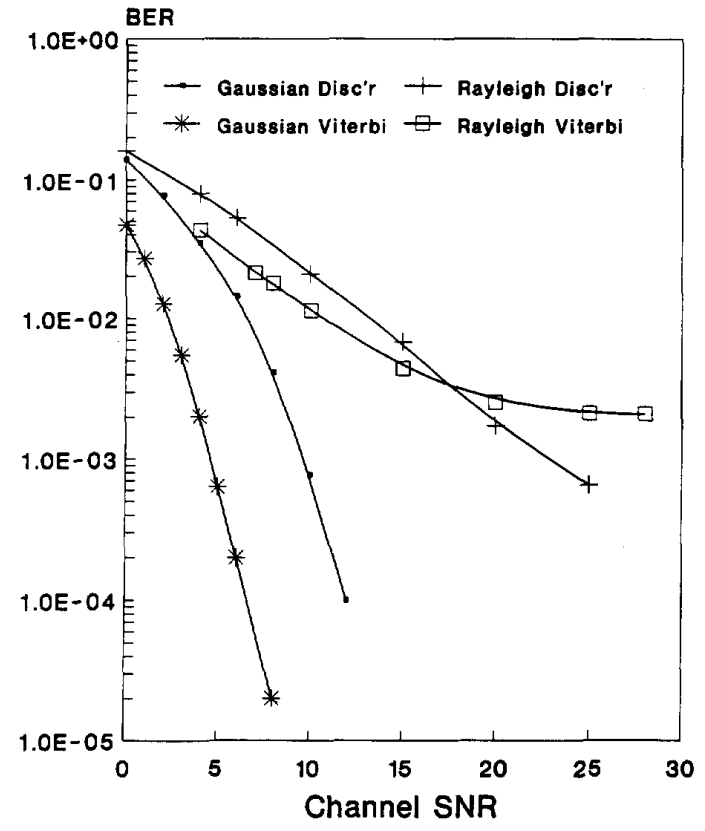

Fig. 2. BER versus SNR performance of GMSK with BT $=0.5$ over Gaussian and Rayleigh fading channels.

recovery was more difficult due to the smaller openings of the eye-pattern.

The BER performances of our GMSK modems with $B_{T}=$ 0.5 and $B_{T}=0.3$ in the presence of Gaussian and Rayleigh fading channels when using frequency discriminators and Viterbi detection are shown in Figs. 2 and 3, respectively. The receiver filter had a bandwidth equal to the bit rate. The Viterbi detector with both GMSK schemes $\left(B_{T}=0.5\right.$ and $B_{T}=0.3$ ) yielded very similar performances for transmissions over both types of channels. Although the performance of the frequency discriminator was degraded by the reduction in $B_{T}$, to comply with the spectrally tighter GSM and DCS 1800 proposals in our further experiments, we opted for $B_{T}=0.3$. We mention that in the presence of frequency dispersion in wide-band channels and provided the channel estimates are accurate, then the Viterbi detector performance will actually improve because path diversity is inherent in the metric calculation.

2) $\frac{\pi}{4}$-DQPSK Performance: Both the American dual-mode cellular system D-AMPS (IS-54), and the Japanese dualmode cellular system employ $\frac{\pi}{4}$-shifted differential quaternary phase shift keying, with square root raised cosine Nyquist filtering using a roll-off factor of 0.35 at both transmitter and receiver. This appears a sensible compromise between the intersymbol interference when the sampling point at the receiver is incorrect, the FIR Nyquist filter length, and the channel separation required. The schemes achieve a modulation spectral occupancy of $1.62 \mathrm{~b} / \mathrm{Hz}$.

The performance of $\frac{\pi}{4}$-DQPSK was evaluated over the Gaussian and Rayleigh fading channels with both differential phase detection as in System D or J and MLH-CR as in System $\mathrm{C}$ or I. The performance of these systems is shown in Fig. 4. With the AWGN channel, the MLH-CR was consistently

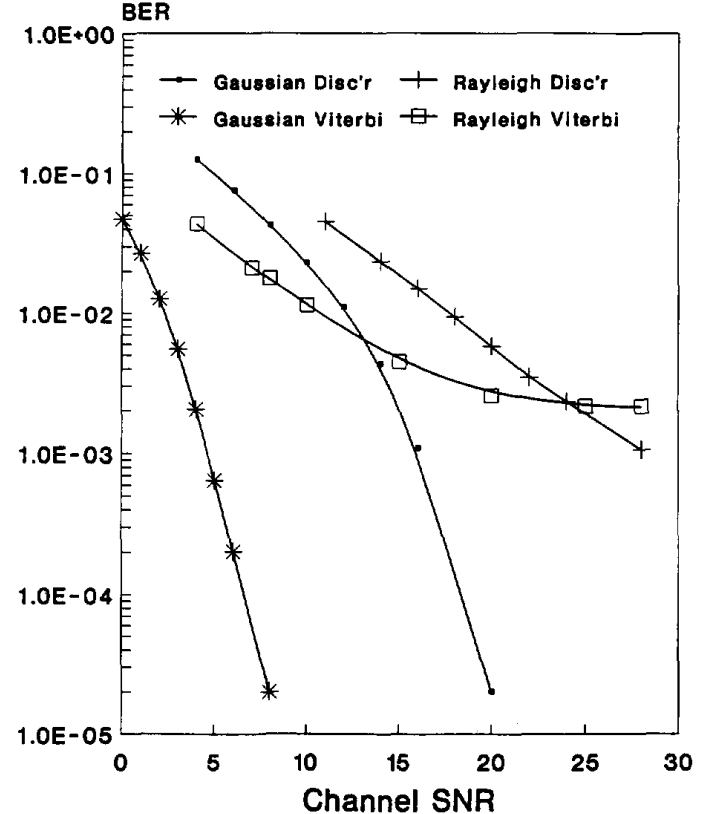

Fig. 3. BER versus SNR performance of the GMSK modem with $B T=0.3$ used in Systems A, B, G and H over Gaussian and Rayleigh fading channels.

better. For the Rayleigh fading channel, its higher complexity was hardly justifiable for channel SNR's below $15 \mathrm{~dB}$, which was deemed to be the designed operating range of the DQPSK modem. At lower fading rates, the MLH-CR performance would improve, as the estimates of the channel attenuation and phase shift would more closely reflect the actual instantaneous channel behavior between updates of the signal estimates, i.e., the channel would appear to be more stationary.

3) 16-StQAM Performance: In this scheme, both a differential arrangement similar to that in [10], as well as an MLH-CR were deployed, relating to Systems $\mathrm{F}$ and $\mathrm{E}$ or $\mathrm{L}$ and $\mathrm{K}$, respectively. The channel attenuation and phase shift were estimated using channel sounding every 60 symbol periods. The BER performance of these schemes in terms of channel SNR is portrayed in Fig. 5. System F or $\mathrm{L}$ with the differential detector performed worse over the Gaussian channel, but for the Rayleigh fading channel, it exhibited no residual BER over our range of channel SNR values. If the Rayleigh fading rate was increased due to either a higher mobile velocity, a higher transmission frequency, or a lower symbol rate, the channel estimates would become more inaccurate and the MLH-CR performance of System $\mathrm{E}$ or $\mathrm{K}$ would deteriorate more rapidly than for the differential scheme of System $F$ or $L$.

4) Modem Performance Comparison: Our findings from Figs. 2-5 are summarized as follows. The GMSK modem with the complex Viterbi detector had significantly higher robustness than the multilevel schemes for transmissions over Gaussian channels. This conspicuous advantage disappeared when a low complexity discriminator was used, or when the Rayleigh channel was present, since the low normalized bandwidth of $B_{N}=0.3$ was associated with a fairly closed eye diagram, rendering discriminator-based decisions 


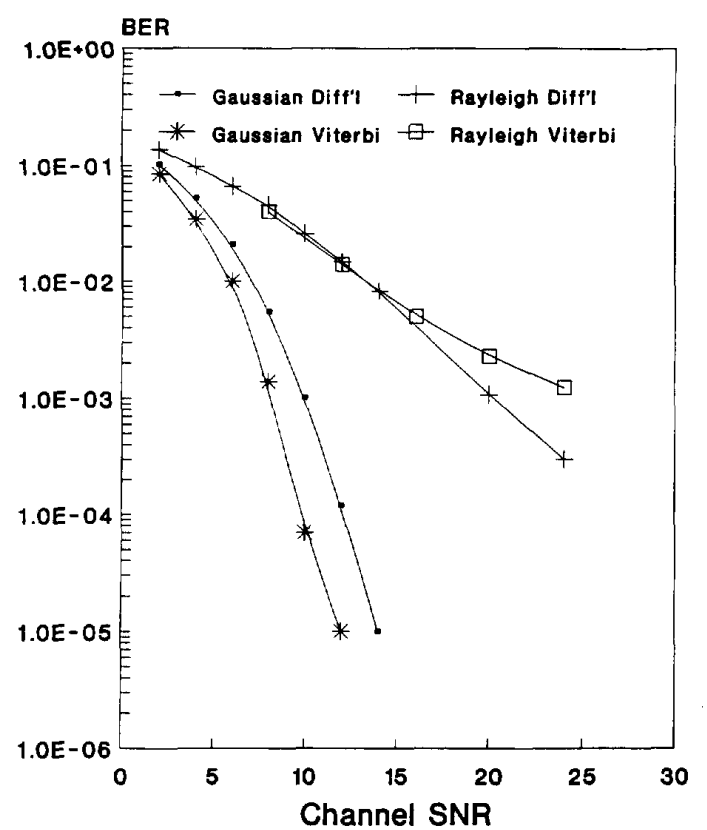

Fig. 4. BER versus SNR performance of the $\frac{\pi}{4}$-DQPSK modem used in Systems C, D, I and J over Gaussian and Rayleigh fading channels.

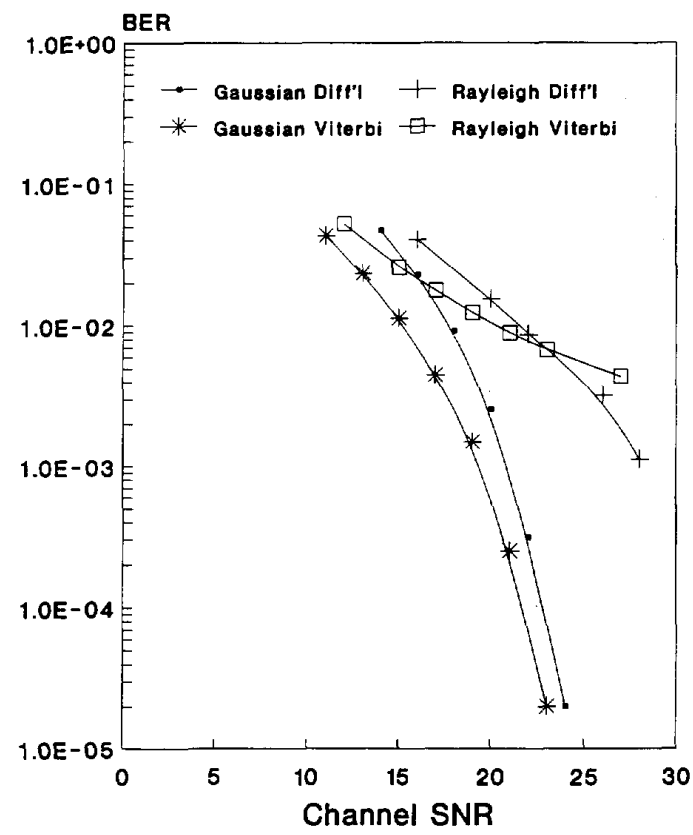

Fig. 5. BER versus SNR performance of the "16-StQAM modem used in Systems E, F, K and L over Gaussian and Rayleigh fading channels.

prone to error. Hence, the quaternary $\frac{\pi}{4}$-DQPSK scheme outperforms this low complexity binary scheme, providing a further interesting system optimization trade-off. The more complex MLH-CR had a marginally superior performance in the presence of Gaussian channels than did the simple differential detector. However, there was no advantage when the transmissions were over Rayleigh channels. The modems in Systems A-F had similar BER performances in the presence of Rayleigh channels, requiring channel SNR's in excess of about $20 \mathrm{~dB}$ for BER's of approximately $10^{-3}$.

\section{B. Speech System Performance}

We now consider transmitting speech over our modems. The objective performance of our systems was evaluated in terms of decoded speech segmental SNR (SSNR) and cepstral distance degradation (CDD) versus channel SNR for both the Gaussian and the Rayleigh fading channels. The tendencies revealed in terms of SSNR were always confirmed also by the CDD curves, and hence for the sake of econony we only present our findings as regards SSNR. Furthermore, the SSNR versus channel BER at the output of the modulator was plotted to reveal the dependence of speech quality on the statistical error distribution.

1) Cordless Telecommunication Schemes: Cordless telecommunication (CT) systems [3] are typically used in office environments. CT transceivers have low power consumption and low complexity and currently do not deploy FEC codecs. For the schemes A-F in Table I, we employed the low complexity ADPCM codec without the use of FEC coding.

a) GMSK/ADPCM Performance: Let us consider systems $\mathrm{A}$ and $\mathrm{B}$, employing GMSK with $B_{T}=0.3$ and the ADPCM speech codec. In error-free conditions, this speech codec achieved a segmental SNR of $29 \mathrm{~dB}$ with our test speech signal. Fig. 6 reveals that unimpaired speech quality was achieved for the Gaussian channel with the Viterbi detector (System A) for SNR's in excess of about $6 \mathrm{~dB}$, whereas the discriminator (System $B$ ) required about $20 \mathrm{~dB}$, as suggested by the BER curves in Fig. 3. For transmissions over the Rayleigh fading channel, the Viterbi detector never reached the error-free SSNR $=29 \mathrm{~dB}$, since it had an irreducible error rate at high SNR's, as seen in Fig. 3. Despite its poorer modem BER performance at low SNR's, System B did not suffer from an irreducible error rate. Consequently, above 25 $\mathrm{dB}$ channel SNR, its BER and speech SSNR performance was better than that achieved by System A for Rayleigh fading channels. The residual BER of the Viterbi detector of System A can be reduced by sounding the channel more frequently, but this reduces the throughput. For operational channel SNR values, the Viterbi detector's low residual BER can be removed by FEC coding and unimpaired speech performance can be ensured.

It is interesting to observe in Fig. 7 how differently the various channels and detection schemes affect the speech SSNR performance while maintaining the same channel BER. For example, at a channel BER of $4 \times 10^{-3}$, System A provides near perfect speech quality with SSNR $=25 \mathrm{~dB}$ over the Rayleigh fading channel, while the less complex System B has an unacceptably low SSNR of about $5 \mathrm{~dB}$ over the Gaussian channel. Apparently, occasional error bursts inflicting the same average number of errors affect the SSNR performance less objectionably than the more evenly distributed random errors generating continuously impaired speech. These tendencies are also reflected by Fig. 6 . Interestingly, this situation will be 


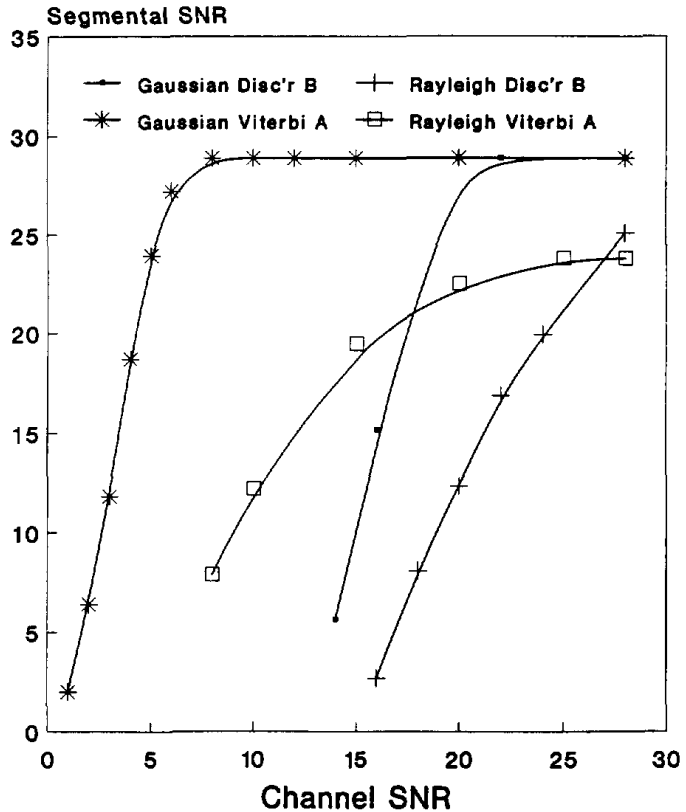

Fig. 6. Speech performance of Systems A and B: SSNR versus channel SNR.

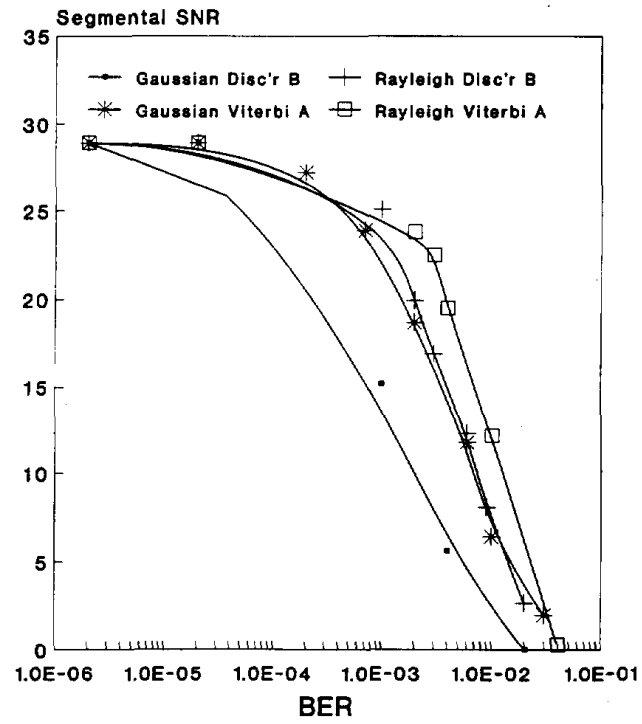

Fig. 7. Speech performance of Systems A and B: SSNR versus channel BER.

reversed when deploying FEC codecs, which can combat the less serious random impairments of Gaussian channels but fail to remove all the error bursts.

The discriminator performed worst over the Gaussian channel, while the Viterbi detector used over the Rayleigh fading channel coped best with the errors. This was because a burst of errors caused less overall SSNR degradation than a continually impaired speech signal. Similar conclusions accrue from the CDD curves not plotted here, although apart from

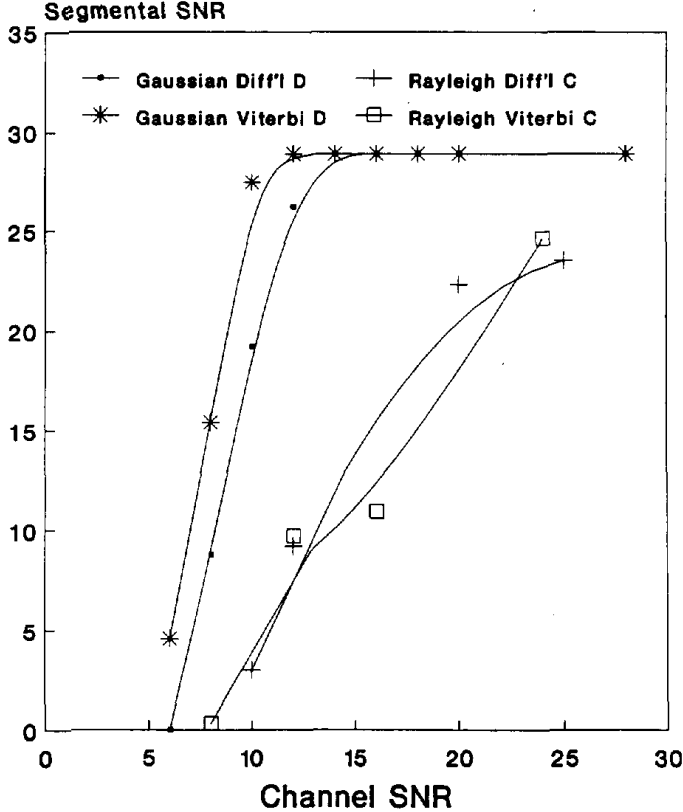

Fig. 8. Speech performance of Systems C and D: SSNR versus channel SNR.

the Rayleigh discriminator performance, the speech quality degradation measured in CDD was less steep for channel SNR's in excess of $15 \mathrm{~dB}$. This fact was confirmed by our experience with objective versus subjective speech quality assessments, ensuring acceptable subjective speech quality in this channel SNR region.

Overall, if low channel SNR operation is crucial, then the higher complexity of the Viterbi detector is outweighed by its better performance. However, above $25 \mathrm{~dB}$ channel SNR, the low-complexity discriminator performs better when transmissions are over Rayleigh fading channels.

b) $\frac{\pi}{4}-D Q P S K / A D P C M$ Performance: Consider Systems $\mathrm{C}$ and $\mathrm{D}$, which incorporated the $\frac{\pi}{4}$-DQPSK modulator with either MLH-CR or a differentially coherent detector, respectively, combined with an unprotected ADPCM speech codec. The objective speech quality of these schemes is shown in Fig. 8. The more complex MLH-CR offered slightly better BER and SSNR performances for transmissions over the Gaussian channel, a trade-off not necessarily worthwhile. Similar to the GMSK/ADPCM arrangement, the CDD exhibited a less steep curve than the SSNR degradation, a fact that correlated with our informal perceptual impressions. For Rayleigh fading channels, the two detectors performed similarly.

In a system with a lower symbol rate, where the fading envelope fluctuates with respect to the symbol period more rapidly, the MLH-CR performance will be degraded. Notice in Fig. 9 that assuming the same average channel BER, the random distribution of errors in the Gaussian channel degraded' the SSNR of the recovered speech more drastically than the error bursts from the Rayleigh fading channel. The differential detector performed equally well as the complex MLH-CR.

c) 16-StQAM/ADPCM Performance: Systems E and F employed the Star 16-QAM modulator in conjunction with 


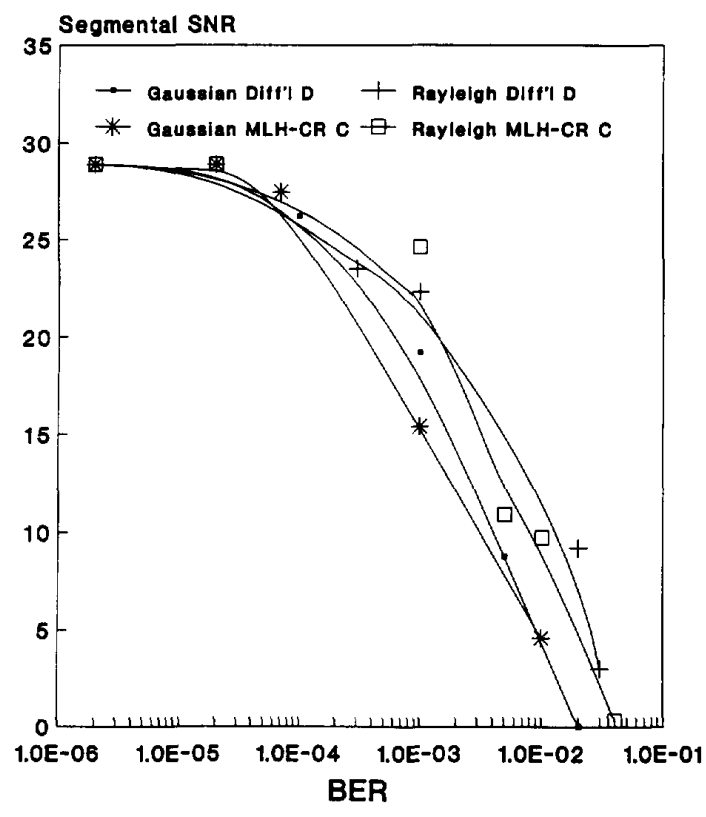

Fig. 9. Speech performance of Systems C and D: SSNR versus channel BER.

ADPCM speech coding. The performance in terms of SSNR versus channel SNR is given in Fig. 10. In this multilevel scheme, the probability of error was marginally different for each of the four bits in the symbol. Each ADPCM sample was coded as four bits. Each of these bits had a different significance, so the speech coder output symbols had to be correctly mapped onto the modulator symbols for optimum performance. For toll quality speech with ADPCM transmissions, a channel BER $<10^{-3}$ is required. This condition was satisfied for channel SNR's $>20 \mathrm{~dB}$ in the case of the MLH-CR, but the differential detector requires some 2 $\mathrm{dB}$ higher SNR for transmissions over the AWGN channel. For the Rayleigh fading channel, the MLH-CR's BER curve tended to a residual error rate (see Fig. 5) and this corresponded to the poor improvement in SSNR with increasing channel SNR, as shown in Fig. 10. The performance of the differential detector was better for channel SNR's $>22 \mathrm{~dB}$ and this BER curve exhibited no residual value.

Overall, the differential demodulator was more attractive due to its lower complexity and achieved a similar or better BER performance than that of the MLH-CR. Similar to the performances obtained with the GMSK and DQPSK modems, the variation of SSNR with channel BER in Fig. 11 indicated that the burstiness of the Rayleigh fading channel was more favorable than the evenly distributed errors associated with the Gaussian channel. Again, with less frequent channel estimation, faster fading, or lower symbol rate, the performance of the MLH-CR would be further degraded. Thus, the differential detector, with its advantage of low complexity, is more appropriate for operation with Rayleigh fading channels.

d) Comparison of CT Performance: From our assessment of systems using the ADPCM speech codec, we conclude

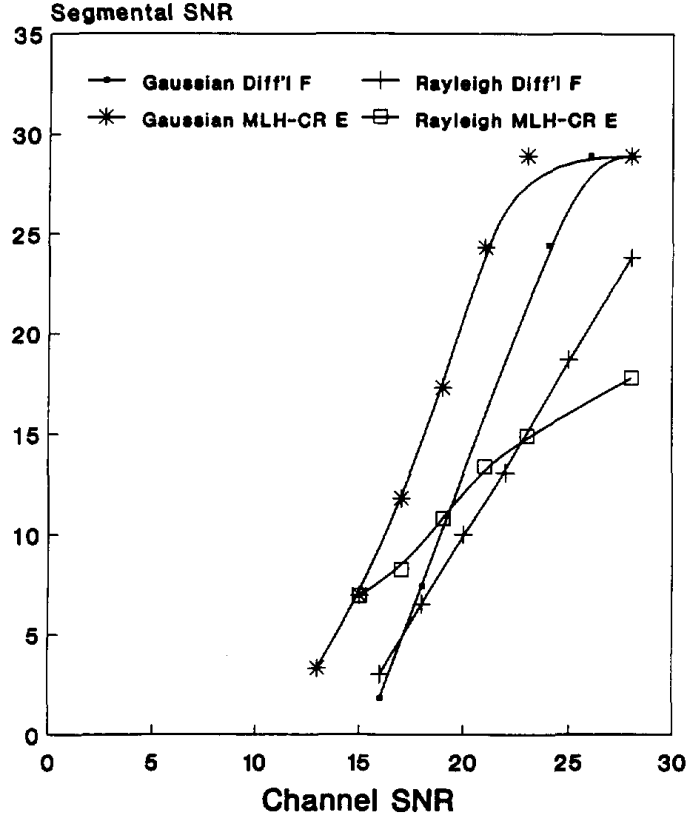

Fig. 10. Speech performance of Systems E and F: SSNR versus channel SNR.

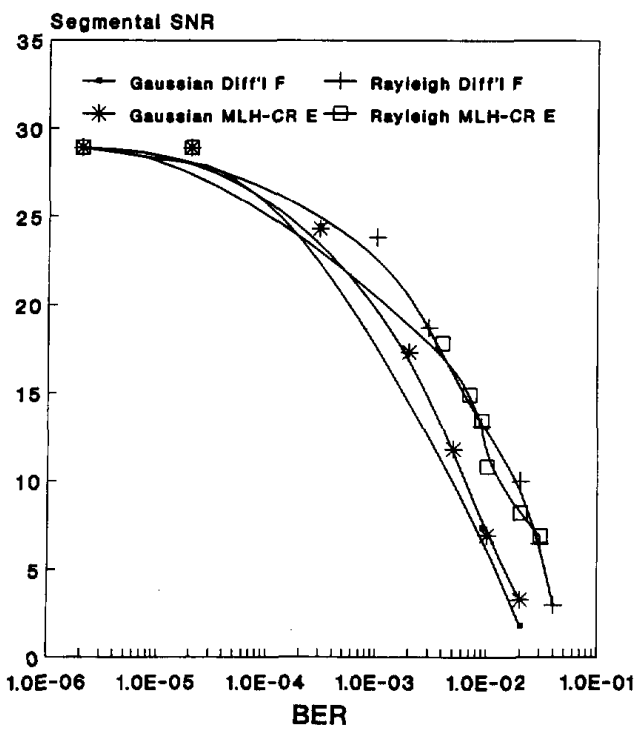

Fig. 11. Speech performance of Systems E and F: SSNR versus channel BER.

that, for the Gaussian channel, the GMSK system offers the best performance in terms of SNR but has the lowest throughput. Doubling the number of bits per symbol by moving to the DQPSK scheme, sacrifices $5 \mathrm{~dB}$ in channel SNR. Changing to Star QAM, with four bits per symbol, results in a further $12 \mathrm{~dB}$ penalty. For high channel SNR values, the lower complexity frequency discriminator and differential decoder schemes are preferable to the more complex MLH-CR. This is because they have similar, or 


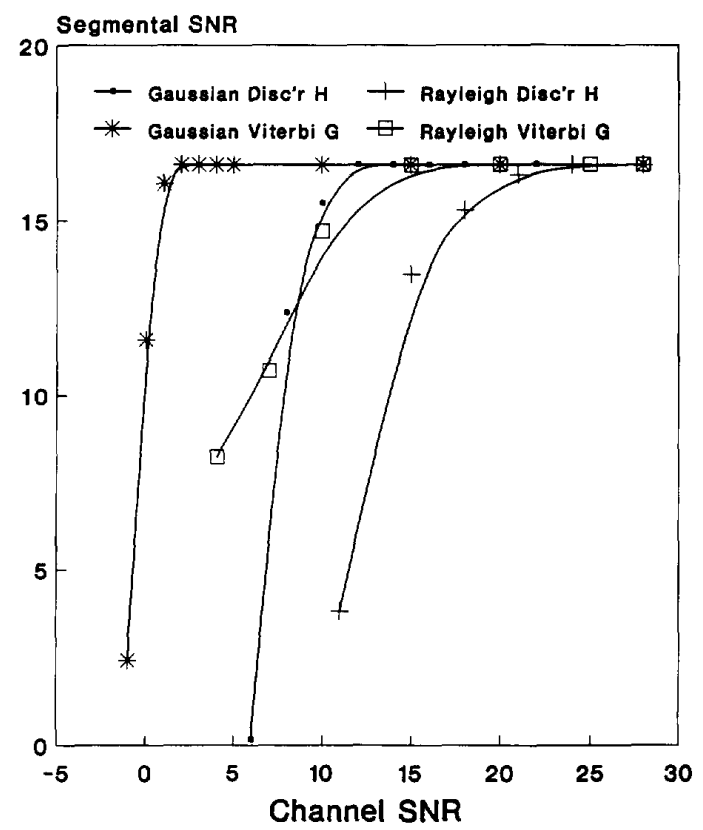

Fig. 12. Speech performance of Systems $G$ and H: SSNR versus channel SNR.

for high channel SNR values, superior BER, SSNR and CDD performances.

In harmony with the modem performances evaluated, the Viterbi decoded GMSK/ADPCM scheme had an impressive performance with the benign Gaussian channel. However, if the channel was fading, the less complex discriminator provided better speech quality. For the multilevel modems, the low complexity differential approach was preferred. A minimum of about $25 \mathrm{~dB}$ channel SNR was required for unprotected, unimpaired speech quality, a strong justification in favor of the use of high bandwidth-efficiency multilevel modem schemes. The minimum required channel SNR values based on both SSNR and CDD curves are summarized in columns 13 and 14 of Table I, although due to lack of space this treatise does not provide CDD curves. Therefore, there are occasional slight differences between the channel SNR values seen from the SSNR curves and those of Table I.

2) Robust Systems: We now move on to consider the more sophisticated schemes with FEC coding and the RPE-LTP speech coder. Again, the GMSK, $\frac{\pi}{4}$-DQPSK and 16-StQAM modems were used, each with either a simple or a complex detector.

a) GMSK/BCH/RPE Schemes: Systems $\mathrm{G}$ and $\mathrm{H}$ consisted of a BCH-coded RPE-LTP speech codec in conjunction with a GMSK modem using either Viterbi detection or a frequency discriminator. Because of the FEC, the SSNR reached the error-free unimpaired condition at high channel SNR's for all the scenarios, as shown in Fig. 12. The poor performance of the frequency discriminator was apparent. (With a BT value of 0.3 , its performance was seriously degraded by the extra ISI compared to $\mathrm{BT}=0.5$.) The Viterbi detector required some $10 \mathrm{~dB}$ less channel SNR than the

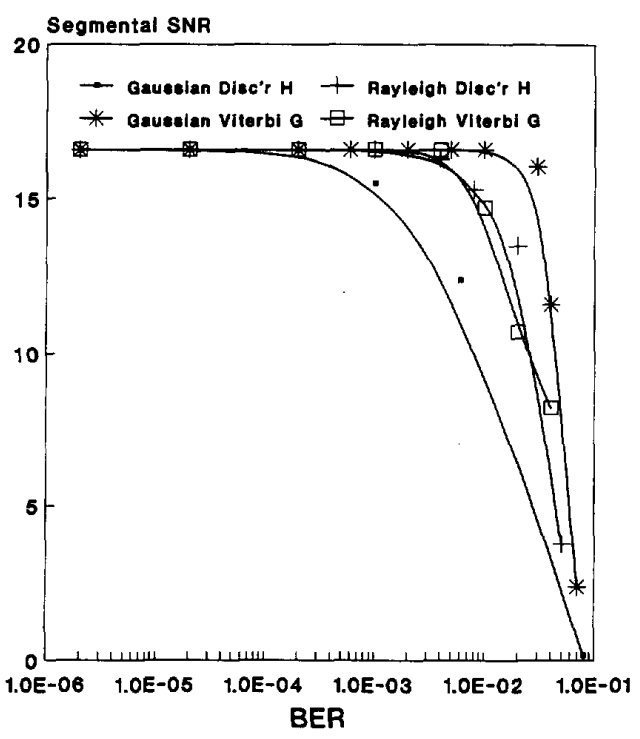

Fig. 13. Speech performance of Systems G and H: SSNR versus channel BER.

frequency discriminator for both types of channels for an unimpaired SSNR of about $16 \mathrm{~dB}$.

Examining the SSNR versus channel BER curves of Fig. 13 reveals that, while the error distributions over the Rayleigh fading channel are similar for the outputs of the two demodulators, the performance over the Gaussian channel was vastly different. The Viterbi detector output was well matched to the FEC such that near error-free performance was achieved with a raw channel bit error rate of $3 \%$. The discriminator operating over the Gaussian channel appeared to have an extremely inconvenient systematic distribution of errors, causing the FEC to be frequently overloaded. Surprisingly, this meant that the bursty errors over the Rayleigh channel were more easily accommodated. Thus, the Viterbi detector was the most appropriate demodulator for this scheme, as shown in Fig. 13.

b) $\frac{\pi}{4}$-DQPSK/BCH/RPE Schemes: Systems I and J incorporated RPE-LTP speech coding, BCH FEC and DQPSK with either MLH-CR or differential detection. Fig. 14 shows that the SSNR objective speech performance of the two schemes was very similar. This was expected from the BER versus channel SNR modem performances seen in Fig. 4, where we preferred the lower complexity differential detector. The MLH-CR had less than a $2 \mathrm{~dB}$ advantage for the AWGN channel, while the differential detector had the edge when the Rayleigh fading channel was used. Fig. 15 shows that the two detectors had similar channel error patterns, and that the best speech quality was obtained for an error rate $<1 \%$ over the Gaussian channel. The overloading of the FEC in the Rayleigh fading channel occured for both Systems I and J, and BER $<0.1 \%$ was required to achieve peak SSNR and best CDD performance. Overall, the less complex differential detector was favored.

c) 16-StQAM/BCH/RPE Schemes: The SSNR versus channel SNR performance of Systems $\mathrm{K}$ and $\mathrm{L}$ is shown 


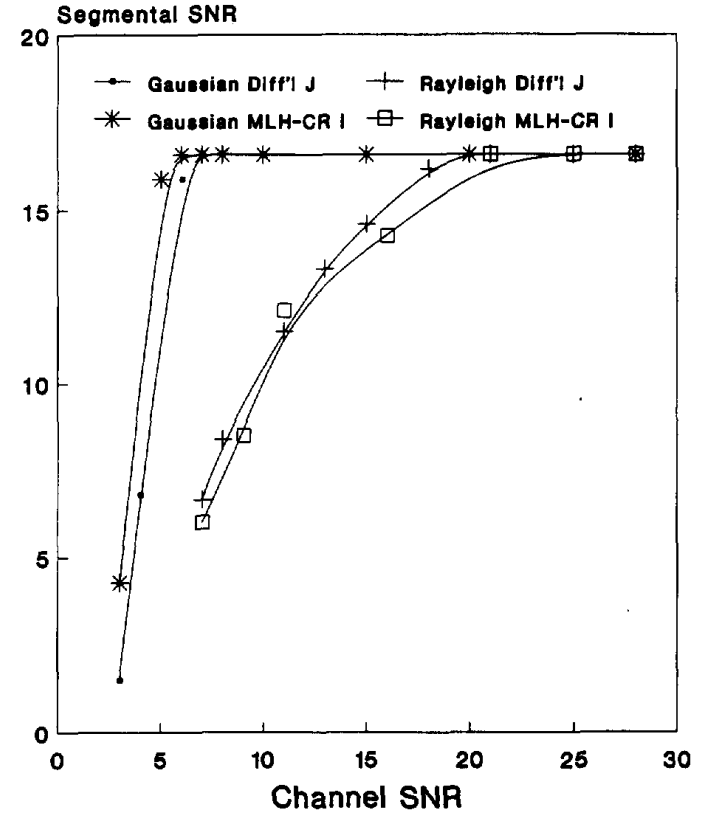

Fig. 14. Speech performance of Systems I and J: SSNR versus channel SNR.

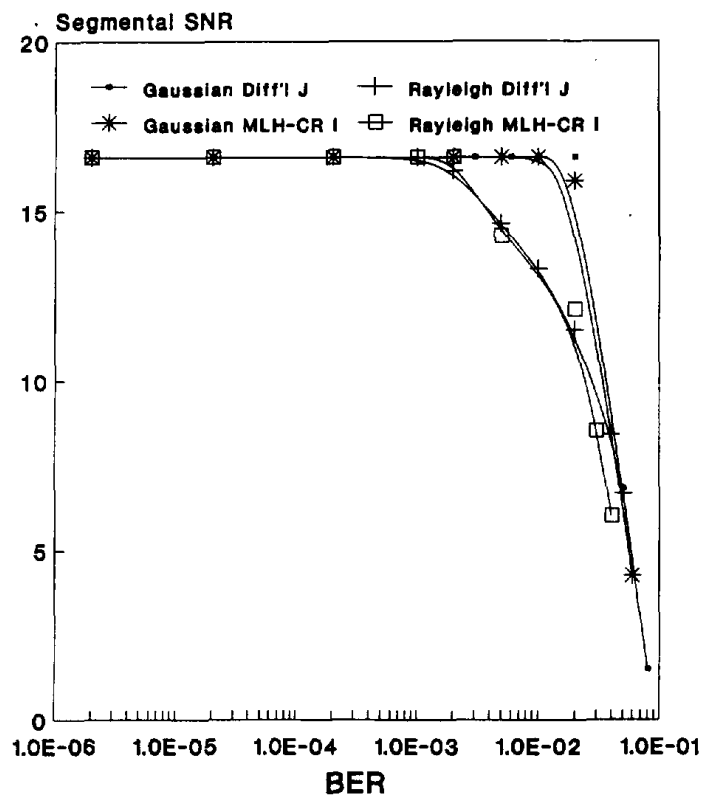

Fig. 15. Speech performance of Systems I and J: SSNR versus channel BER.

in Fig. 16. These systems consisted of the RPE-LTP speech codec, BCH channel coding and 16-level "Star" QAM detected by either the MLH-CR or the differential detector. In terms of BER versus channel SNR modem performance depicted in Fig. 5, we favored the differential detector due to its lower complexity and lack of residual BER for transmissions via fading channels. When using FEC coding, the residual BER associated with the MLH-CR detector was effectively removed

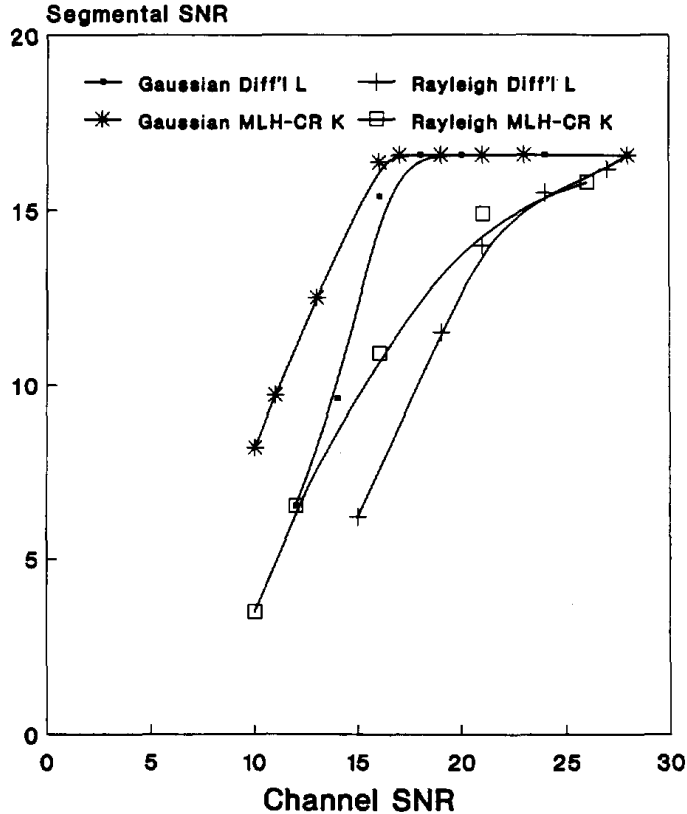

Fig. 16. Speech performance of Systems $K$ and L: SSNR versus channel SNR.

and the systems with differential or MLH-CR detection had similar overall SSNR and CDD performances for both Gaussian and Rayleigh channels. However, the complexity advantage of the differential scheme made it more attractive. The SSNR versus BER curve in Fig. 17 shows that, at the same channel BER, the SSNR speech quality was lower over the Rayleigh fading channel than over the Gaussian one. This is in contrast to our experiences with the unprotected CT schemes where the SSNR speech performance over the Rayleigh channel was usually better at the same channel BER than the Gaussian channel's speech SSNR performance. The reason for this phenomenon is that the FEC codec removes most of the less serious random errors, but often fails under bursty errors. The MLH-CR and differential detectors behaved similarly in this respect.

d) Robust System Performance Comparison: As anticipated, the BCH-protected RPE-LTP schemes were more robust. For the GMSK modem, the Viterbi detector was very attractive for both the channels we investigated, requiring a mere $10 \mathrm{~dB}$ channel SNR for unimpaired speech quality in the presence of Rayleigh fading. For the multilevel modem, the simpler differential detector was preferred. These $\mathrm{BCH}-$ protected systems removed the modem's residual BER, yielding essentially unimpaired, error-free speech quality, which was rarely achieved without FEC coding via fading channels for unprotected CT. The more robust, higher quality speech transmissions are attainable at a channel coded rate of $24.8 \mathrm{kbps}$, instead of the unprotected and less complex 32 kbps ADPCM transmissions, a trade-off available to system designers. Over nonfading Gaussian channels, typically $5 \mathrm{~dB}$ lower channel SNR was sufficient for similar speech quality when using the FEC-protected robust schemes, assuming 


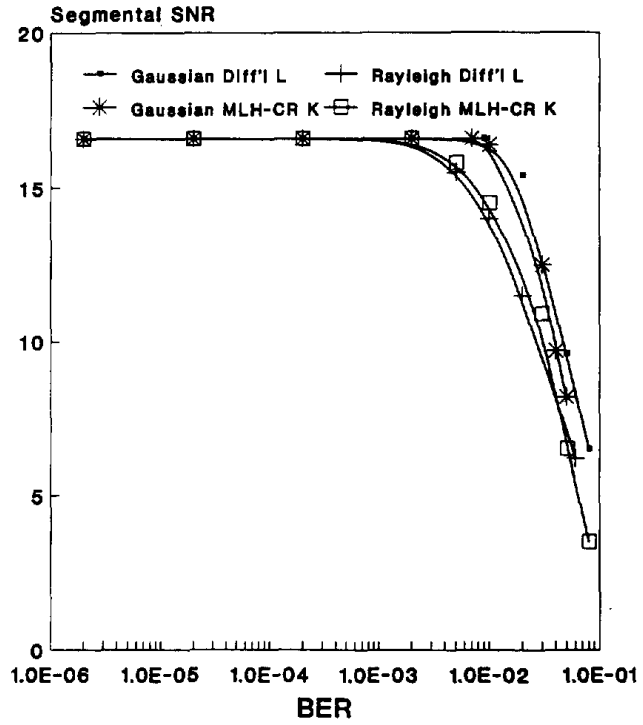

Fig. 17. Speech performance of Systems $\mathrm{K}$ and L: SSNR versus channel BER.

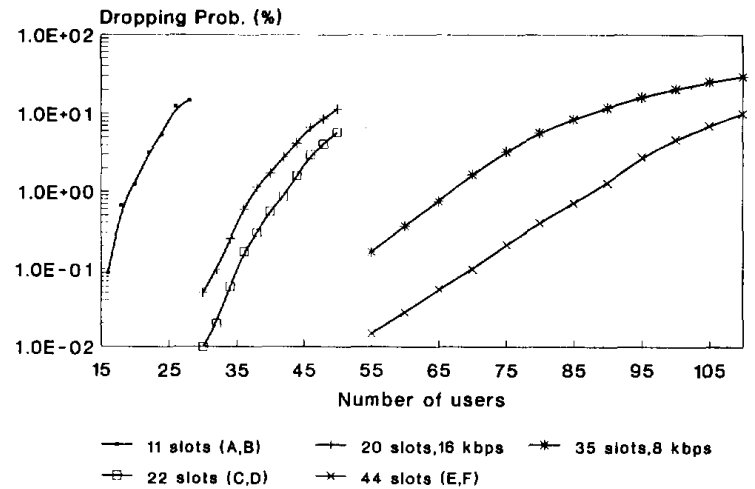

Fig. 18. Packet dropping probability versus number of users without FEC.

identical modems. Again, our results are summarized in columns 13 and 14 of Table I.

\section{PRMA Performance}

PRMA efficiency was evaluated in terms of packet dropping probability $\left(P_{d r o p}\right)$ versus number of users for Systems A-L, using the number of slots listed in column 9 of Table $I$. Our results are shown in Figs. 18 and 19 for the unprotected and FEC-coded scenarios, respectively. For comparison, we also evaluated the equivalent packet dropping curves for hypothetical $16 \mathrm{kbps}$ half- and $8 \mathrm{kbps}$ quarter-rate speech codecs, which are represented by the curves " 20 slots, $16 \mathrm{kbps}$ " and " 35 slots, $8 \mathrm{kbps}$ " in Fig. 18.

Considering Fig. 18, observe that at $P_{d r o p}=1 \%$ about five more users were served, when using a $2 \mathrm{~b} / \mathrm{symbol}$ modem combined with a $32 \mathrm{kbps}$ speech codec as in System C or D, which supported approximately 42 conversations, than in

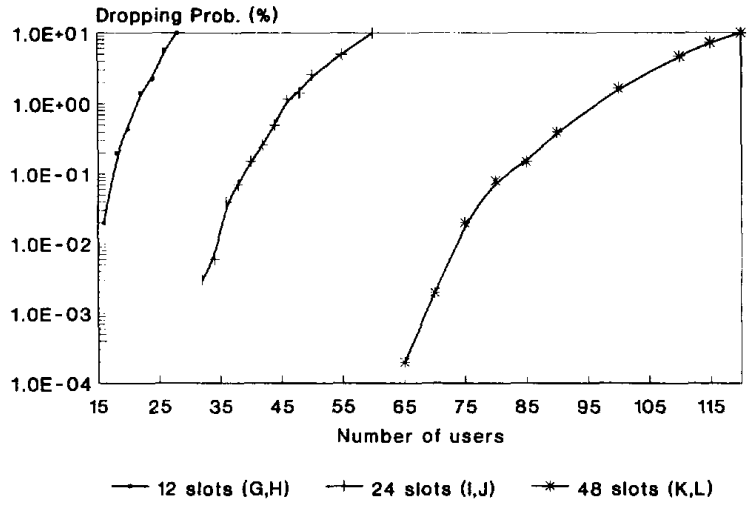

Fig. 19. Packet dropping probability versus number of users with FEC.

the case of an identical Baud-rate hypothetical system using a $16 \mathrm{kbps}$ speech codec and binary modem scheme, which supported 20 slots and serviced about 37 users. Furthermore, the lower complexity was also associated with the former scheme. This phenomenon became even more pronounced when we compared a hypothetical $8 \mathrm{kbps}$ speech codec and binary modem combination serving some 67 subscribers with the $32 \mathrm{kbps}$ codec and 16-StQAM modem combination of Systems E and F, which supported about 87 users. Explicitly, some 20 more users can be supported at lower associated complexity, when using $4 \mathrm{~b} /$ symbol multilevel QAM modems instead of $8 \mathrm{kbps}$ quarter-rate speech codecs. The number of PRMA users supported by Systems A-L is summarized in column 10 of Table $\mathbf{I}$.

The relative number of PRMA users per TDMA time slot for the 32, 16 and $8 \mathrm{kbps}$ speech codecs combined with binary transmissions is computed with the aid of Fig. 18 using the number of users supported at $1 \%$ dropping probability as $18 / 11=1.64,37 / 20=1.85$ and $67 / 35=1.91$ users $/$ slot, respectively. As seen from columns 9 and 10 of Table $I$, the corresponding figures for the 1,2 , and $4 \mathrm{~b} /$ symbol modem, $32 \mathrm{kbps}$ speech codec combinations are $18 / 11=1.64,42 / 22$ $=1.91$ and $87 / 44=1.98$, respectively, emphasizing the higher efficiency of the systems with multilevel modems. This fact is further accentuated by their less steep dropping probability curves, yielding a more benign overloading characteristic and higher grade of service.

As seen from Fig. 19, similar tendencies are valid for our FEC-protected RPE-LTP schemes as well. The number of users supported in case of the 1, 2, and 4 b/symbol Systems G-L is 22, 46 and 96, respectively. The associated PRMA efficiences are expressed using columns 9 and 10 of Table I as $22 / 12=1.83,46 / 24=1.92$ and 2.18 users/slot, respectively. The relative number of PRMA users/slot values are listed in column 11 of Table I. The normalized PRMA user bandwidth figures listed in column 12 of Table I can be derived by dividing the TDMA user bandwidth values in column 8 by the relative number of PRMA users/slot given in column 11 of Table I. The normalized PRMA bandwidth figures vary between the extreme values of 14.5 and 4.7 , emphasizing again the superiority of the multilevel schemes. 


\section{IMPLICATIONS OF OUR EXPERIMENTS}

In our discourse, we have examined a number of radio links formed with different subsystems, some of which are to be found in the second generation cordless telecommunication (CT) systems of CT2 and DECT, and in the cellular systems of GSM, DCS 1800 and IS-54. We consider that third generation systems will need backward compatibility with these second generation systems, and therefore, third generation transceivers will contain in firmware formats subsystems that can be reconfigured under software control to emulate the second generation transceivers, as well as those of the more advanced UMTS.

Faced by a myriad of alternatives, we restricted ourselves to 12 different links that contained three modulators, four demodulators, one FEC codec and two speech codecs. Our approach was not to arrive at a definitive system, as this requires many man years of effort, rather we sought to offer an approach to a set of scenarios and to make deductions that will be useful. To make the problem more tractable, we considered the provision of mobile telephony in microcells. Summaries of our findings were given in Sections VII-A-4, VII-B-1-d, VIIB-2-d and in Table I, while Figs. 1-19 provided our detailed results in graphical form.

System designers prefer to operate with a robust system, which for our subsystems suggests the binary GMSK modulator using a simple frequency discriminator and the ADPCM speeh codec. This arrangement works well in CT as the channel SNR can be designed to be sufficiently high to give low BER's. If the number of users requiring radio access increases, the $\frac{\pi}{4}$-DQPSK modem could be used, and with even greater demand, the 16-StQAM would be preferred, assuming in both cases that the increases in channel SNR can be accommodated. The increase in SNR required relative to GMSK is 5 and $17 \mathrm{~dB}$ for $\frac{\pi}{4}$-DQPSK and Star QAM, respectively. If the channel SNR can be sufficiently high, the frequency discriminator and differential decoder schemes are preferred. For the ADPCM codec, the Baud rate decreases from 32 to 16 to 8 as the number of bits per Baud handled by the modem increases. The effective bandwidth required by each user decreases correspondingly from 23.7 to 19.8 to $13.3 \mathrm{kHz}$.

As described in Section VI, we arrange for the ADPCM encoded speech bits to be formed into packets along with appropriate headers. The resulting bit rate becomes $35.2 \mathrm{~kb} / \mathrm{s}$. Arranging for a PRMA frame duration of $20 \mathrm{~ms}$ and for our $400 \mathrm{kBd}$ rate, there are 11 TDMA time slots when GMSK is used. As we move to multilevel modulation, the size of the packet in bits remains unaltered but the packet size in terms of symbols decreases. When there are four bits per Baud (Star QAM), the Baud rate is decreased by four times, and as we may equate packet length with slot size, the number of slots becomes 44 . From Fig. 18, we see that 87 users can be supported with a $1 \%$ dropping probability. The ability to almost double the number of TDMA slots is due to the ability of the PRMA scheme to exploit the silence periods in the speech conversations.

When channel SNR's are relatively low, then the options to use $\frac{\pi}{4}$-DQPSK and Star QAM are unavailable if extra capacity is required, although with GMSK and ADPCM the deployment of PRMA increases the number of TDMA slots per carrier from 11 to 18 . However, by using an RPE-LTP speech codec in combination with a BCH FEC codec results in 12 TDMA time slots, and hence the number of PRMA users increases to 22. By using the Viterbi detector, this arrangement can operate with a channel SNR of $10 \mathrm{~dB}$ in a Rayleigh fading channel, and only $3 \mathrm{~dB}$ over Gaussian channels.

When the channel SNR's are increased to 6 or $16 \mathrm{~dB}$ for Gaussian or Rayleigh channels, respectively, the DQPSK modem can be used in conjunction with PRMA to support 46 users within 24 TDMA slots per carrier. If conditions improve such that the channel SNR's exceed 16 or $24 \mathrm{~dB}$ for Gaussian and Rayleigh channel conditions, the Star QAM can be deployed. When PRMA is added, 96 PRMA users per carrier can be accommodated within the 48 TDMA slots available.

We now imagine an adaptive transceiver having all the subsystems listed in Table I. Whenever possible, the PRMA system uses the GMSK modem, the ADPCM speech codec and no channel coding to maintain high system capacity and robustness, while retaining low power consumption.

The number of modulation levels and other features of the modulator and demodulator are programmable. The modem consumes the least power in its binary mode, and when it uses the simple frequency discriminator.

As channel conditions worsen, or as the offered teletraffic increases, the modulation and the demodulation change appropriately. The simple ADPCM codec is replaced by the more power consuming combination of RPE-LTP and BCH codecs, and PRMA is used. In our systems listed in Table I, for a packet dropping probability of $1 \%$, the range of the number of PRMA users per slot can be changed from 1.64 to 2.18 by adapting the transceiver's parameters.

In such a short discourse, we have been limited by the number of scenarios that could be examined. However, it is apparent that given particular channel SNR's and offered teletraffic, then it would be desirable if the transceiver adapted itself to minimize the power consumption from its battery while maintaining imperceptible speech degradations.

In the same way, as the modem should be designed to reconfigure itself under software control, so must the speech and FEC codecs. Ideally, these codecs should be embedded types with their outer FEC codecs being switched in to enhance performance under adverse conditions. If not, the unused codecs at any moment of time should be "dormant," taking minimal power, while most of the power is assigned to the active codecs.

Fig. 1 shows the concept of our adaptive transceiver. The speech encoder, bit mapper, channel coder interleaver, and modulator are adapted to channel conditions and teletraffic demand. The PRMA slot allocator assigns slots depending on both system control and the voice activity detector (VAD). The VAD also controls the speech codec, varying its bit rate to suit the speech input and the ambient acoustics. When a person stops speaking, the bit rate is reduced to encode the essential background sounds. The channel codec also adapts, applying different power FEC's dependent on the sensitivity 
of the speech bits. The interleaver works in conjunction with the diversity arrangement and depends on the depth and rate of fading.

The receiver essentially reverses the processes performed at the transmitter. In addition, it monitors the channel quality by noting the performance of the channel decoder. This quality factor is relayed by the BS to the mobile to control its configuration. We also include in Fig. 1 a postenhancement system [9] to statistically remove the effect of errors when the FEC decoding has failed. Future mobile radio transceivers are likely to have considerable intelligence built into them in order to combat a variety of conditions and to be backward compatible with second generation networks. We trust that our discussions based on a limited number of experiments and our wider ranging remarks will stimulate further work in this area.

\section{REFERENCES}

[1] E. Bacs and L. Hanzo, "A simple real-time adaptive speech detector for SCPC systems," in Proc. ICC '85, Chicago, IL, May 1985, pp. $1208-1212$.

[2] S. Nanda, D. J. Goodman and U. Timor, "Performance of PRMA: A packet voice protocol for cellular systems," IEEE Trans. Veh. Technol., vol. 40, no. 3, pp. 584-598, Aug. 1991.

[3] W. H. W. Tuttlebee, Ed., Cordless Telecommunications in Europe. London: Springer-Verlag, 1990.

[4] R. Steele, Mobile Radio Communications. New York: IEEE Press-London: Pentech Press, 1992.

[5] R. V. Cox, J. Hagenauer, N. Seshadri and C. E. W. Sundberg, "Subband speech coding and matched convolutional channel coding for mobile radio channels," IEEE Trans. Signal Processing, vol. 39, no. 8, pp. 1717-1731, Aug. 1991

[6] H. Shi, P. Ho and V. Cuperman, "A combined CELP/Reed-Solomon codec for mobile radio applications," in Proc. IEEE 42nd VTC'92, Denver, CO, May 10-13, 1992, pp. 187-191.

[7] H. Suda and T. Miki, "An error protected $16 \mathrm{~kb} / \mathrm{s}$ voice transmission for land mobile radio channel," IEEE J. Select. Areas Commun., vol. SAC-6, no. 2 , pp. 346-352, 1988.

[8] K. Feher, "MODEMS for emerging digital cellular-mobile radio system," IEEE Trans. Veh. Technol, vol. 40, no. 2, pp. 355-365, May 1991.

[9] L. Hanzo, R. Steele and P. M. Fortune, "A subband coding, BCH coding and 16-QAM system for mobile radio communication," IEEE Trans. Veh. Technol., vol. 39, no. 4, pp. 327-340, Nov. 1990.

[10] W. T. Webb, L. Hanzo and R. Steele, "Bandwidth efficient QAM schemes for Rayleigh fading channels," IEE Proc. Part I, vol. 138, no. 3, pp. 169-175, June 1991 .

[11] W. Webb, L. Hanzo, R. A. Salami and R. Steele, "Does 16-QAM provide an alternative to a half-rate GSM speech codec?" in Proc. IEEE Veh. Technol. Conf., St. Louis, MO, May 19-22, 1991, pp. 511-516.

[12] G. Larson, B. Gudmundson and K. Raith, "Receiver performance for the North American digital cellular system," in Proc. IEEE-VTC, St. Louis, MO, May 19-22, 1991, pp. $1-6$.

[13] M. Ikura, K. Ohuo, Y. Yamao and F. Adachi, "Field experiments on TDMA mobile radio signal transmissions," IBID, pp. 669-674.

[14] K. Murota and K. Hirade. "GMSK modulation for digital mobile radio telephony," IEEE Trans. Commun., vol. 29, pp. 1044-1050, July 1981.

[15] M. K. Simon and C. C. Wang, "Differential detection of Gaussian MSK in a mobile radio environment," IEEE Trans. Veh. Technol., vol. 33, no. 4, pp. 307-320, Nov. 1984

[16] D. Divsalar and M. K. Simon, "Multiple-symbol differential detection of MPSK,"IEEE Trans. Commun., vol. 38, no. 3, pp. 300-308, Mar. 1990.

[17] T. Suzuki and T. Mizuno, "Multiple-symbol differential detection for differential amplitude modulation," submitted to Int. Zurich Seminar ' 94.

[18] D. J. Goodman, "Cellular packet communications," IEEE Trans. Commun., vol. 38, no. 8, pp. 1272-1280, Aug. 1990.

[19] N. Kitawaki, M. Honda, and K. Itoh, "Speech-quality assessment methods for speech-coding systems," IEEE Commun. Mag., vol. COM22 , no. 10 , pp. $26-32$, Oct. 1982.

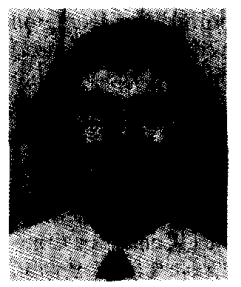

John E. B. Williams received the B.Eng. in electronic engineering with first class honors from Southampton University in 1989.

In 1985, he joined Rolls-Royce Plc as an undergraduate apprentice. In 1989, he completed his training at Rolls-Royce, Bristol, and was appointed a Technology Engineer in the Control Systems and Accessories Department. He joined Multiple Access Communications Limited in 1990 as an Engineer. While at MAC Ltd., he has been working on a three-year research project investigating the "intelligent multimode terminal." This project has involved extensive computer simulations, including the simulation of speech codecs, channel codecs, fading channels, various modulators and multiple access techniques, and teletraffic. In writing the software for these simulations, he has gained an in-depth knowledge of the $\mathrm{C} / \mathrm{C}++$ programming languages.

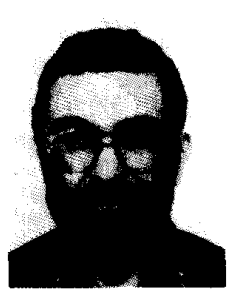

Lajos Hanzo (M'91-SM'92) graduated with a first class Dipl.Ing. electronics degree in 1976 and in 1983 he was conferred the Ph.D. degree with distinction.

During his 18 year career in telecommunications, he has held various research and academic posts in Hungary, Germany and the UK. Since 1986, he has been with the Department of Electronics and Computer Science, University of Southampton, UK and has been a Consultant to Multiple Access Communications Limited, UK. He coauthored two books on mobile radio communications, Mobile Radio Communications (IEEE Press-Pentech Press) and Modern Quadrature Amplitude Modulation (IEEE Press-Pentech Press), published over 70 research papers and was awarded a number of distinctions.

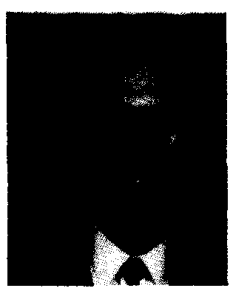

Raymond Steele (SM'80) received a B.Sc. degree in electrical engineering from Durham University, England, in 1959 and Ph.D. and D.Sc. degrees from Loughborough University of Technology, England, in 1975 and 1983, respectively.

Before attaining the B.Sc., he was an indentured Apprentice Radio Engineer. After research and development posts with $\mathrm{E} \mathrm{K}$ Cole, Cossor Radar and Electronics, and Marconi, he joined the lecturing staff at the Royal Naval College, London. He moved to Loughborough University in 1968 where he lectured and directed a research group in digital encoding of speech and picture signals. During the summers of 1975, 1977 and 1978, he was a Consultant to the Acoustics Research Department at Bell Laboratories in the USA, and in 1979, he joined the Company's Communications Methods Research Department, Crawford Hill Laboratory, NJ, USA. He retumed to England in 1983 to become Professor of Communications in the Department of Electronics and Computer Science at the University of Southampton, a post he retains. From 1983 to 1986 , he was a nonexecutive Director of Plessey Research and Technology and from 1983 to 1989 he was a Consultant to British Telecom Research Laboratories. In 1986, he formed Multiple Access Communications Limited, a company concerned with digital mobile radio systems. He is the author of Delta Modulation Systems (New York: Halstead, 1975), editor of Mobile Radio Communications (London: Pentech Press, 1992), and editor of a series of books on digital mobile communications. He has been a conference and session organizer of numerous intemational conferences and a keynote speaker at many international meetings. $\mathrm{He}$ is also Senior Technical Editor of the IEEE Communications Magazine.

Dr. Steele and his coauthors were awarded the Marconi Premium in 1979 and in 1989, and the Bell System Technical Journal's Best Mathematics, Communications, Techniques, Computing and Software and Social Sciences Paper in 1981. He is a Fellow of The Royal Academy of Engineering and a Fellow of the IEE in the UK. 


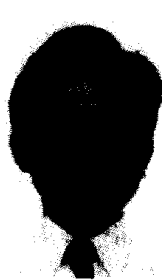

Joseph C. S. Cheung was bom in Hong Kong on November 21, 1966. He received the B.Sc. (Hons.) degree in electrical and electronic engineering from the University of Manchester Institute of Science and Technology (UMIST), England, in 1988 and a Ph.D. degree from the University of Southampton, England in 1992. His Ph.D. thesis studied the design of adaptive equalizer for mobile radio channels.

Upon graduation, he joined the Center for Communications Research at the University of Bristol,

England. His research interests are digital signal processing, modeling and performance evaluation of mobile communication systems. 